\title{
The Association Between Drug Approvals and Burden of Disease From 1990 to 2017
}

J Gen Intern Med 36(11):3615-7

DOI: $10.1007 /$ s11606-020-06525-3

(C) Society of General Internal Medicine 2021

\section{BACKGROUND}

This study examines the correlation between burden of disease and pharmaceutical innovation in the USA - the most profitable commercial market for pharmaceuticals. The paper examines whether certain major disease categories receive more attention from pharmaceutical companies and if the investment portfolio of pharmaceutical companies changed between 1990 and 2017.

\section{METHODS}

There are many ways to measure the investment in research and development by pharmaceutical companies. We measure the result of pharmaceutical research and development using FDA approval of New Molecular Entities (NMEs). In choosing this metric, we recognize that some investments have not resulted in NMEs and that some NMEs are more clinically significant than others.

We obtained all NMEs (small molecules and biologics) approved from 1990 to $2017 .{ }^{1}$ We used the primary pathological indication for approval to categorize each NME into a disease category. US burden of disease was measured using disability-adjusted life years (DALYs) ${ }^{2}$ The correlation between NMEs and DALYs was calculated using a Pearson correlation coefficient. We measured the association between the changes in DALYs and NMEs between the first (1990 1999) and last (2008-2017) decade. We performed the analysis including and excluding orphan drugs.

\section{RESULTS}

There were 866 NMEs approved by FDA between 1990 and 2017. The correlation between NMEs and DALYs was 0.51 (Fig. 1). Five disease categories (neoplasm, infection, endocrine, circulation, and nervous disorders) were responsible for $66 \%$ of NMEs but represented only $45 \%$ of the DALYs. Disease categories with high levels of DALYs but relatively low levels of drug approvals were mental illness, musculoskeletal, respiratory, congenital, and perinatal disorders. Categories of diseases with higher levels of drug approvals than would be predicted based on DALYs alone were neoplasms, infectious diseases, and endocrine disorders.

Between the 1990-1999 and 2008-2017 periods, neoplasm drugs showed the greatest increase in number of NMEs, while circulatory disease experienced the greatest decrease in NMEs. The change in the number of NMEs for the remaining diseases was close to the line predicted by the change in DALYs (Fig. 2).

\section{DISCUSSION}

The pharmaceutical industry concentrated its research and development investments in five disease categories that represent less than half of all DALYs between 1990 and 2017. Mental illness is responsible for the greatest percentage of DALYs but had significantly fewer NMEs than would be predicted by DALYs alone. Neoplasms experienced a significant increase in relative number of NMEs when comparing the 1990-1999 period to the 20082017 period. Similar conclusions were reached when using total deaths as a measure of burden of disease.

Drug companies have no obligation to invest in areas with the greatest burden of disease. Their choice of investment can be a function of the state of science, FDA rules, and the payment system for different types of drugs. Because it takes time to 


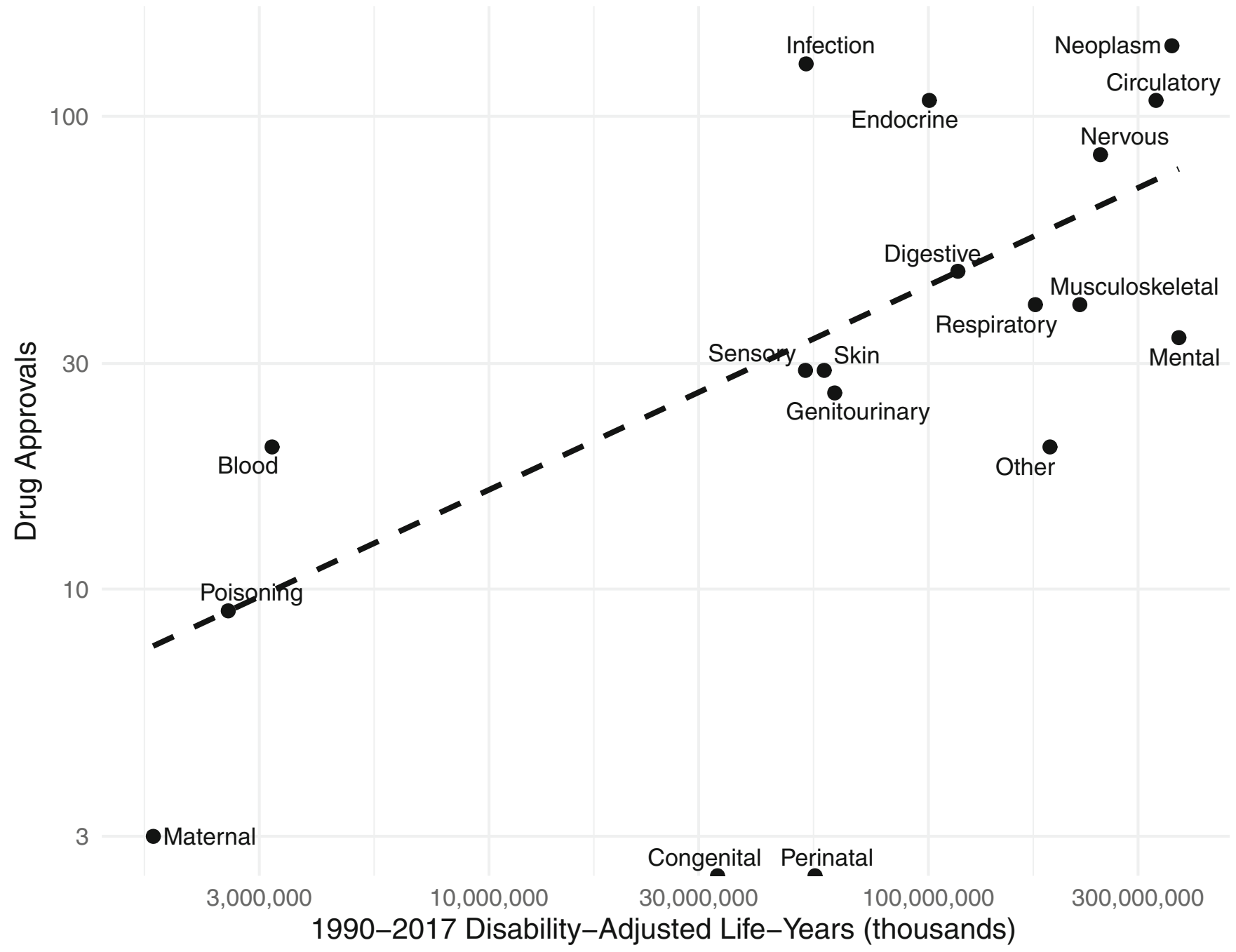

Figure 1 Drug approvals and burden of disease (1990-2017).

develop a drug, there can be a significant lag between NME approval and research and development investment. While not a perfect measure of investment, NMEs represent research and development outcomes, and when these priorities do not represent diseases with the greatest burden, it suggests certain diseases receive less attention. An important question, therefore, is whether the incentives should be modified. This question is particularly salient given the important role public funding, vis-à-vis NIH, has in establishing the basic science that leads to the research centers, venture capitalists, and ultimately the pharmaceutical industry developing new drugs. ${ }^{3}$ The apparent mismatch between initial investments in research and development and the ultimate development of NMEs could be modified with FDA rules and payment systems if they are found to discourage investment in certain disease categories. Further research is needed to explore whether the mismatch in funding priorities is addressable through policy action. 


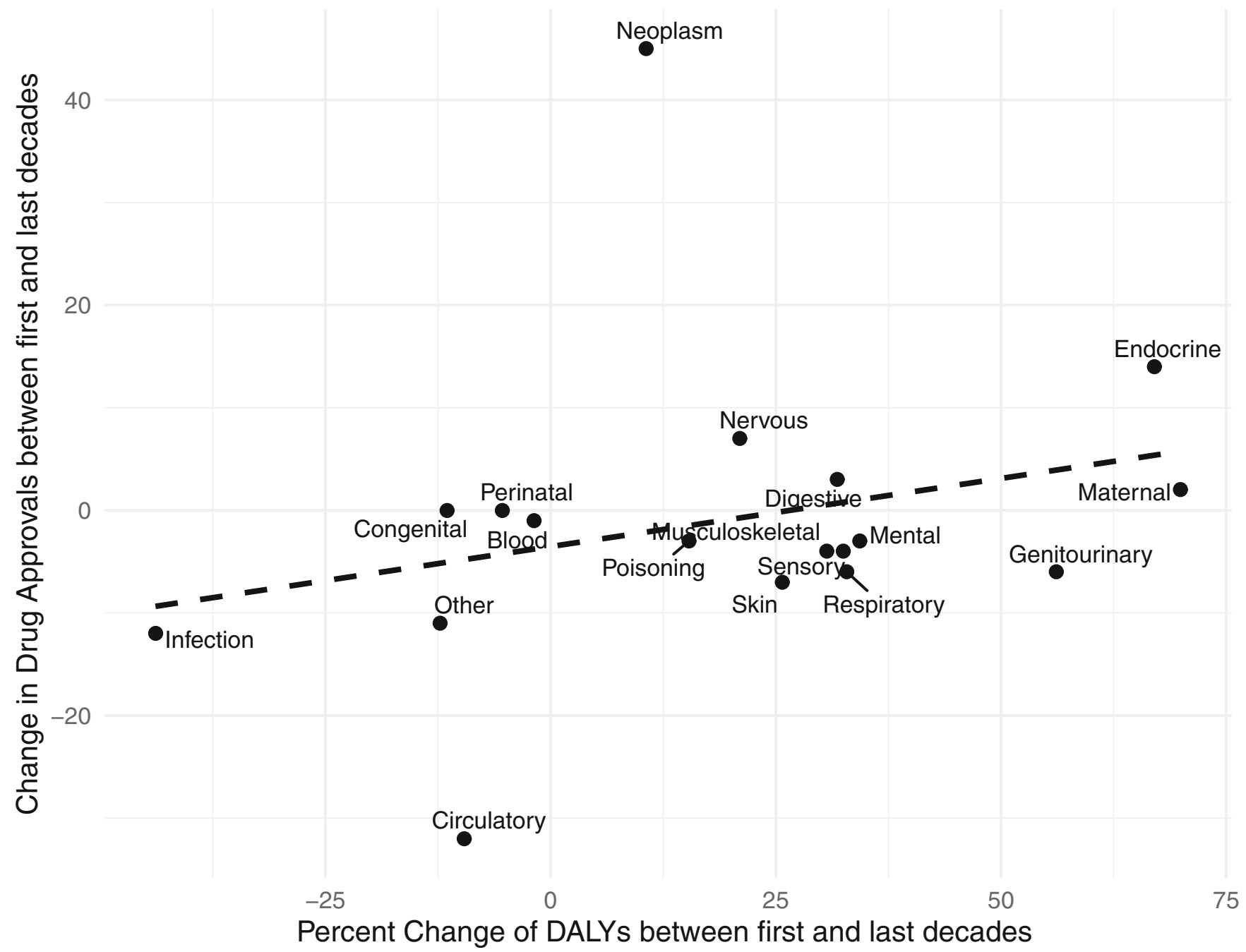

Figure 2 Changes in DALYs and NMEs between the first and last decade.

Tarun George, MD, MHS ${ }^{1}$

Jeromie Ballreich, $\mathrm{PhD}, \mathrm{MHS}^{2}$

Antonio Trujillo, $\mathrm{PhD}^{1}$

Gerard Anderson, $\mathrm{PhD}^{2}$

${ }^{1}$ Department of International Health, Johns Hopkins Bloomberg School of Public Health,

Baltimore, MD, USA

${ }^{2}$ Department of Health Policy and Management, Johns Hopkins Bloomberg School of Public Health, Baltimore, MD, USA

Corresponding Author: Jeromie Ballreich, PhD, MHS; Department of Health Policy and Management, Johns Hopkins Bloomberg School of Public Health, Baltimore, MD, USA (e-mail: jballre2@jhu.edu).

\section{Compliance with Ethical Standards:}

Conflict of Interest: The authors declare that they do not have a conflict of interest.

\section{REFERENCES}

1. Research C for DE and. Compilation of CDER New Molecular Entity (NME) Drug and New Biologic Approvals. FDA [Internet]. 2020 Feb 21 [cited 2020 Jul 29]; Available from: https://www.fda.gov/drugs/drug-approvals-anddatabases/compilation-cder-new-molecular-entity-nme-drug-and-new-biologic-approvals

2. Global Burden of Disease Study 2017. Institute for Health Metrics and Evaluation (IHME). 2018.

3. Cleary, E. G., Beierlein, J. M., Khanuja, N. S., McNamee, L. M., \& Ledley, F. D. (2018). Contribution of NIH funding to new drug approvals 2010-2016. Proceedings of the National Academy of Sciences, 115(10), 2329-2334.

Publisher's Note Springer Nature remains neutral with regard to jurisdictional claims in published maps and institutional affiliations. 